\title{
COMUNICACIÓN
}

\section{Infección por Toxocara canis en población adulta sana de un área subtropical de Argentina}

\author{
JOSÉ M. ALONSO*, MARÍA DE LOS A. LÓPEZ*, MARÍA V. BOJANICH* y JORGE MARULL*

\begin{abstract}
Toxocara canis INFECTION IN ADULT HEALTHY POPULATION FROM A SUBTROPICAL AREA IN ARGENTINA
\end{abstract}

Toxocarosis is a sanitary problem extended world-wide. Numerous reports dealing with this subject have been published in Argentina, focusing many of them about its impact on human health. However, as toxocarosis is not a disease of compulsory notification and many cases are asymptomatic, actual prevalence rates are unknown and the disease is not recognised as a public health problem. The aim of this work was to determine the extent of the infection among healthy adult population from a subtropical region of Argentina, where high prevalence in children have been previously reported. For this purpose, we selected 355 sera of blood donors (325 men-30 women) aged 18-68 years, from a private-owned blood bank of Resistencia, a city located in Northeast Argentina. All samples tested negative for the infectious diseases routinely investigated for blood donation. Elisa test employing excretion/secretion antigens of larvae $L_{2}$ Toxocara canis was performed and indeterminate cases were confirmed by Western blot. Out of 355 sera, 138 tested positive (127 men-11 women) which means a global prevalence of $38.9 \%$. There was not significant difference in relation to gender $39.1 \%$ for men - 36.7\% for women). The results are consistent with the urban and environmental characteristics of the study area and with socio-cultural habits of its inhabitants, and indicate a strong presence of the ascarid with an important degree of impact on the population.

Key words: Toxocarosis, Visceral Larva migrans, Seroepidemiology, Prevalence.

\section{INTRODUCCIÓN}

La característica casi genética del hombre de vivir rodeado de animales domésticos, ha facilitado que la toxocarosis sea una saprozoonosis helmíntica ampliamente difundida en todo el mundo ${ }^{1}$.

La infección humana por Toxocara canis se produce por la ingestión de huevos embrionados a partir del contacto con suelos infectados, por geofagia, por manos mal lavadas, por onicofagia y en menor proporción por el consumo de vegetales contaminados ${ }^{3}$. También ha sido descripta la infección a partir de carnes poco cocinadas procedentes de huéspedes paraténicos ${ }^{2}$ y recientemente se demostró que el contacto directo con el pelaje de perros infectados podría constituir una vía válida de adquisición de la infección ${ }^{4}$. La transmisión de larvas infectantes por otras vías como la hemática o la

\footnotetext{
* Departamento de Inmunología - Instituto de Medicina Regional Universidad Nacional del Nordeste - Resistencia (Argentina). Av. Las Heras 7273500 - Resistencia - Argentina.

E-mail:jalonso@bib.unne.edu.ar
} 
transplacentaria, aun cuando fueron evaluadas, no pudieron ser demostradas 5 .

Las cifras de seroprevalencia en niños y en adultos muestran una distribución cosmopolita con amplias variaciones de acuerdo a las características sanitarias de cada país o región. Las poblaciones de bajo nivel socio-económico presentan generalmente altas tasas de infección por Toxocara y concomitantemente también alta frecuencia de enteroparásitos; en los países desarrollados, aun cuando la prevalencia de enfermedades parasitarias es habitualmente baja, la toxocariois es la helmintiasis mas frecuente ${ }^{6}$.

En Argentina, se han publicado muchos trabajos sobre el tema, destacando la importancia de esta infección por su impacto en las poblaciones. Sin embargo, por tratarse de una patología que no es de notificación obligatoria y por la existencia de casos asintomáticos, las cifras reales de prevalencia no son bien conocidas y por ello la toxocarosis tiene un bajo reconocimiento como problema de salud pública ${ }^{7}$. Estudios previos señalan que la infección y la enfermedad por Toxocara canis entre los niños de la ciudad de Resistencia (Nor-Este de Argentina) es frecuente y que la contaminación de los suelos está presente ${ }^{8,9}$. Estas circunstancias nos indujeron a ampliar el conocimiento sobre la dimensión de la infección humana por este agente en el área mencionada, para lo cual se decidió determinar la seroprevalencia en una muestra de población adulta sana.

\section{MATERIAL Y MÉTODOS}

En base a una prevalencia esperada del $5 \%$, con nivel de confianza del $95 \%(n=202)$ se estudiaron 355 sueros de donantes de sangre de ambos sexos (325 hombres y 30 mujeres), con edades comprendidas entre los 18 y 68 años, que concurrieron a un Banco de Sangre privado de la ciudad de Resistencia (Nor-Este de Argentina). Se seleccionaron solo los sueros que tuvieron resultados negativos en todos los exámenes serológicos habituales para la donación de sangre (Hepatitis B, Hepatitis C, Enfermedad de Chagas, Sífilis, Brucellosis e infecciones por virus HIV y HTLV).

Se efectuó enzimoinmunoensayo (Prueba de ELISA) con antígenos de excresión/secresión de larvas $\mathrm{L}_{2}$ de $T$. canis (TES) para la búsqueda de anticuerpos de clase Ig G, de acuerdo a la técnica descripta ${ }^{10}$. Los sueros fueron ensayados por duplicado a la dilución 1:100, las DO leídas a 450 $\mathrm{nm}$ y los resultados se expresaron en unidades arbitrarias $\mathrm{R}$ (densidad óptica de la muestra/ densidad óptica del cut-off), considerándose positivos los sueros con valores de $\mathrm{R}>1,2$.

Para el cálculo del cutt-off se seleccionaron previamente 50 individuos adultos de la misma región geográfica ( 25 hombres - 25 mujeres), con los siguientes criterios de inclusión:

- Buen estado general de salud

- Ausencia de signos/síntomas clínicos compatibles con toxocarosis: linfoadenopatías, dolor abdominal, hepatopatías, asma, bronquitis, alteración visual.

- Valores de Ig E total < $50 \mathrm{UI} / \mathrm{dl}$

- Eosinofilia $<$ de 3\%.

Se efectuó el test de ELISA por duplicado en todos estos sueros, se calculó el valor promedio de las densidades ópticas y se consideró como valor del cutt-off al promedio +2 DS.

En los casos en los que el test de ELISA arrojaba valores de $\mathrm{R}$ entre 1,0 y 1,2 los sueros fueron considerados "indeterminados" y reestudiados mediante Western blot, empleando los mismos antígenos TES, aceptando como criterio de positividad la presencia de bandas de 24 a 35 $\mathrm{kD}^{11}$.

\section{RESULTADOS}

Aplicando el criterio de unidades R establecido se encontró que de los 355 sueros estudiados, 128 fueron positivos, correspondiendo a 117 hombres y 11 mujeres. También se obtuvo 14 sueros con valores de DO considerados indeterminados, de los que se confirmaron 10 como positivos, con lo que finalmente se determinó una prevalencia global de infección del 38,9\% (138/ $355)$.

No se encontró diferencia significativa entre hombres $(127 / 325=39,1 \%)$ y mujeres $(11 / 30=$ $36,7 \%$ ). Atendiendo a que el muestreo correspondió íntegramente a población adulta, no se consideró de interés discriminar los valores de positividad según franjas etarias.

\section{DISCUSIÓN}

La toxocarosis es una de las infecciones helmínticas mas frecuentemente reportadas en el mundo. Los estudios de prevalencia efectuados 
entre adultos sanos de áreas urbanas de países desarrollados señalan valores diversos, como ser $2 \%$ en Francia ${ }^{12}, 3,9 \%$ en Italia ${ }^{13}, 7 \%$ en Suecia ${ }^{14}$, y valores más elevados en pobladores rurales, como ser $16 \%$ en Escocia ${ }^{15}$ o un $37 \%$ en el sur de Francia donde se considera endémica a esta patología ${ }^{16}$.

En los países en desarrollo, particularmente en los de climas cálidos, las cifras de prevalencia son generalmente altas, pudiendo alcanzar valores extremos como ser $92,8 \%$ entre los adultos de la isla de La Reunión ${ }^{17}$.

Existen numerosas publicaciones sobre variados aspectos de esta infección originadas en Argentina; sin embargo, las referencias sobre prevalencia en población sana son muy escasas. Una revisión Medline empleando los descriptores "Toxocariasis, seroprevalencia, Argentina", arrojó como únicos resultados una publicación que informa un $10 \%$ de positividad entre donantes de sangre de la ciudad de Gualeguaychú ${ }^{18}$ y un trabajo que señala un $39 \%$ de positividad entre habitantes de la ciudad de La Plata ${ }^{19}$. El valor de prevalencia hallado en nuestra serie es también alto, muy similar al último mencionado, lo que estaría señalando una situación epidemiológica comprometida para la ciudad de Resistencia.

$\mathrm{Ni}$ el contacto directo con perros aún cachorros, ni la tenencia en domicilio de los animales son condiciones suficientes para adquirir una infección por T. canis. Otras circunstancias epidemiológicas deben coincidir para favorecer el riesgo de una infección que se adquiere a partir de un entorno contaminado. Está bien demostrado que el ambiente físico de las ciudades y los hábitos sociales de sus pobladores juegan un rol crucial en la polución de los suelos y en el mantenimiento y la distribución de los huevos infectantes del helminto ${ }^{2}$. Sin embargo, esta cuestión pareciera que permanece aún subestimada en muchas ciudades argentinas.

La influencia de los factores socioeconómicos sobre la ocurrencia de la infección humana ha sido evaluada por diferentes autores, señalándose que las poblaciones de menor nivel presentan altas prevalencias por una mayor contaminación de los suelos motivada en las características de las viviendas y en los hábitos higiénicos y socioculturales de sus habitantes ${ }^{6-20}$. Estos ambientes también favorecerían que el riesgo de infección se presente mayormente en los peridomicilios y no en los espacios verdes de uso público, como ocurre en las áreas céntricas de las grandes ciudades ${ }^{21}$.

Como fuera informado en un trabajo previo de nuestro grupo de trabajo, la población de Resistencia es de un nivel socioeconómico mayoritariamente bajo, la ciudad presenta una amplia área poblada con calles sin pavimentar y por ende sin limpieza diaria, lo que significa una vasta extensión de terreno expuesta a depósitos de heces caninas. Posee un clima subtropical húmedo y hábitos de conducta social en sus pobladores en relación con la sanidad del medio ambiente que favorecen la contaminación de los suelos con huevos de T. canis $^{9}$.

La toxocarosis se detecta mas frecuentemente en niños, ya que sus hábitos higiénicos y su frecuente contacto con animales, constituyen el grupo muy expuesto. Estas circunstancias dan como resultado que en niños de 1 a 14 años de edad de Resistencia, la seroprevalencia alcance un valor de $37,9 \%{ }^{8}$.

Se conoce que la positividad serológica aumenta con la edad debido a la prolongada viabilidad de las larvas en los tejidos humanos y a las posibilidades de reinfección ${ }^{22,23}$. Al haberse evaluado en este trabajo solamente población adulta, entendemos que el valor de prevalencia hallado resulta compatible con todas las circunstancias antes señaladas y estaría señalando una fuerte presencia del ascárido en el área del estudio, con un importante grado de exposición por parte de sus pobladores. Se concluye que las autoridades de salud deberían ejercer un mayor control sanitario de los perros a fin de prevenir y disminuir la contaminación ambiental, así como promover en la comunidad el concepto de "propiedad responsable" de las mascotas.

\section{RESUMEN}

La toxocarosis es un problema sanitario presente en todo el mundo. Sin embargo, por tratarse de una patología que no es de notificación obligatoria y por la existencia de casos asintomáticos, las cifras de prevalencia real en Argentina no son bien conocidas y por ello la toxocariasis tiene un escaso reconocimiento como problema de salud pública. El objetivo del presente trabajo fue determinar la seroprevalencia en adultos sanos de una ciudad subtropical del NorEste de Argentina, en la que estudios previos indican que la infección infantil por Toxocara 
canis es frecuente y que la contaminación de suelos está presente. Se estudiaron 355 sueros de donantes de sangre (325 hombres y 30 mujeres), con edades entre 18 y 68 años, de un Banco de Sangre privado de la ciudad de Resistencia. Se efectuó enzimoinmunoensayo empleando antígenos de excresión/secresión de larvas $\mathrm{L}_{2}$ de $T$. canis confirmándose mediante Western blot. De los 355 sueros estudiados, 138 fueron positivos (127 hombres y 11 mujeres), lo que significa una prevalencia global de infección del 38,9\%; no se encontró diferencia significativa en relación con el género $(39,1 \%$ en hombres $36,7 \%$ en mujeres). Como el muestreo correspondió íntegramente a población adulta, no se consideró de interés discriminar los valores de positividad según franjas etarias. Los resultados encontrados resultan compatibles con las características urbanas y ambientales del área del estudio y con los hábitos socio-culturales de sus habitantes, y señalan una fuerte presencia del ascárido, con un importante grado de exposición por parte de sus pobladores.

\section{REFERENCIAS}

1.- DESPOMMIER D. Toxocariasis: clinical aspects, epidemiology, medical ecology and molecular aspects. Clin Microbiol Rev 2003; 16: 265-72.

2.- KERR-MUIR MG. Toxocara canis and human health. Br Med J 1994; 309: 5-6.

3.- VAZQUEZ TSUJI O, MARTÍNEZ BARBABOSA I, TAY ZABALA J et al. Bol Chil Parasitol 1997; 52: 47-50.

4.- WOLFE A, WRIGHT I P. Human toxocariasis and direct contact with dogs. Vet Rec 2003; 152: 419-22.

5.- TAYLOR MRH. Toxocariasis in Ireland. En: Lewis JM, Maizels RM eds. Toxocara and Toxocariasis. Clinical, epidemiological and molecular perspectives. Brit Soc Parasitol 1993; 71-80.

6.- ANARUMA FILHO F, CHIEFFI P, CORREA C R et al. Human toxocariasis: a seroepidemiological survey in the municipality of Campinas (SP), Brazil. Rev Inst Med trop S Paulo 2002; 44: 303-7.

7.- ALTCHEH J, NALLAR M, CONCA $M$ et al. Toxocariasis: aspectos clínicos y de laboratorio de 54 pacientes. Ann Pediatr 2003; 58: 425-31.

8.- ALONSO J M, BOJANICH M V, CHAMORRO M, GORODNER J O. Toxocara seroprevalence in children from a subtropical city in Argentina. Rev Inst Med trop S Paulo 2000; 42: 235-7.
9.- ALONSO J M, STEIN M, CHAMORRO M C, BOJANICH M V. Contamination of soils with eggs of Toxocara in a subtropical city in Argentina. J Helminthol 2001; 75: 165-8.

10.- GILLESPIE S H. Migraring worms. En: Gillespie SH, Hawkey PM. eds. Medical Parasitology. A practical approach. New York, IRL Press at Oxford University Press 1995, p. 177-82.

11.- MAGNAVAL J F, FABRE R, MAURIERES $P$ et al. Application of the western-blotting procedure for the immunodiagnosis of human toxocariasis. Parasitol Res 1991; 77: 697-702.

12.- MAGNAVAL J F, GLICKMAN L T, DORCHIES P, MORASSIN B. Highlights of human toxocariasis. Korean J Parasitol 2001; 39: 1-11.

13.- GENCHI C, DI SACCO B, GATTI $S$ et al. Epidemiology of human toxocariasis in northern Italy. Parassitología 1990; 32: 313-9.

14.- LJUNGSTROM I, VAN KNAPEN F. An epidemiological and serological study of Toxocara infection in Sweden. Scan J Infect Dis 1989; 21: 87-93.

15.- GIRDWOOD R, SMITH H, BRUCE R, QUINN R. Human Toxocara infection in west of Scotland. Lancet 1978; 17: 1318.

16.- GLICKMAN L T, MAGNAVAL J F, DOMANSKI L M et al. Visceral larva migrans in French adults: a new disease syndrome? Am J Epidemiol 1987; 125 :101934.

17.- MAGNAVAL J F, MICHAULT A, CALON N, CHARLET J P. Epidemiology of human toxocariasis in La Reunión. Trans Roy Soc Trop Med Hyg 1994; 88: 531-3.

18.- MINVIELLE M C, TAUS M R, RAFFO A et al. Seroprevalence of Toxocariasis in blood donors of Gualeguaychú, Argentina. Trans Roy Soc Trop Med Hyg 2000; 94: 373-5.

19.- RADMAN N E, ARCHELLI S M, FONROUGE RD et al. Human Toxocarosis. Its seroprevalence in the city of La Plata. Mem Inst Oswaldo Cruz 2000; 95: 281-5.

20.- HERRMANN N, GLICKMAN L T, SCHANTZ P M et al. Seroprevalence of zoonotic toxocariasis in the United States: 1971-1973. Amer J Epidem 1985; 122 : 890-6.

21.- HOLLAND C, O'CONNOR P, TAYLOR M R H et al. Families, parks, gardens and toxocariasis. Scand J Infect Dis 1991; 23: 225-31.

22.- OVERGAAUW P. Aspects of Toxocara epidemiology: human Toxocarosis. Crit Rev Microbiol 1997; 23: 21531.

23.- FENOY S, CUELLAR C, AGUILA C, GUILLÉN J L. Persistence of immune response in human toxocariasis as measured by ELISA. Int J Parasitol 1992; 22 : 1037-8.

Agradecimientos: Este trabajo fue parcialmente financiado con fondos del Subsidio PI-254 de la Secretaría General de Ciencia y Técnica de la Universidad Nacional del NordesteArgentina. 